\title{
Measurements at the T2K near detector
}

\author{
Lorenzo Magaletti ${ }^{* \dagger}$ \\ INFN Sezione di Bari \\ E-mail: lorenzo.magaletti@ba.infn.it
}

The near detector complex of T2K consists of a set of scintillating tracking detectors spanning the beam axis (INGRID) along with a magnetized detector system with fine-grained tracking and calorimetry (ND280). Together, ND280 and INGRID offer unique opportunities for the study of $\approx 1 \mathrm{GeV}$ neutrino interactions, including the possibility to study the energy dependence of the cross section using the varying energy spectrum resulting from detectors spanning different offaxis angles, and precise kinematic reconstruction and particle identification with which neutrino interactions can be studied in detail. We report on new studies of charged current muon neutrino interactions in both INGRID and ND280 in final states with zero or one charged pion, including coherent scattering leading to pion production, and measurements of the inclusive cross section.

The European Physical Society Conference on High Energy Physics

22-29 July 2015

Vienna, Austria

* Speaker.

${ }^{\dagger}$ on behalf of the T2K collaboration. 


\section{The near detector complex of $\mathrm{T} 2 \mathrm{~K}$}

The near detector complex, described in detail in [1], is located $280 \mathrm{~m}$ downstream of the neutrino production target and consists of the on-axis Interactive Neutrino GRID detector (INGRID) and the $2.5^{\circ}$ off-axis tracking detector, ND280 (Fig. 1). INGRID consists of a $7+7$ identical array
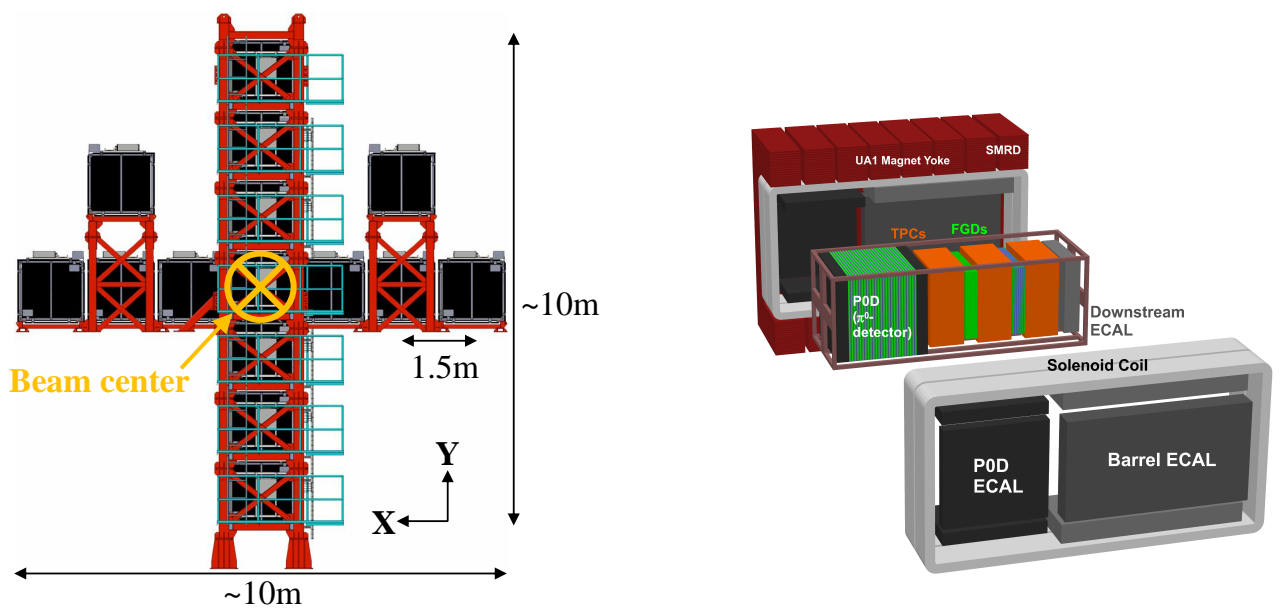

Figure 1: The on-axis near detector INGRID (left) and the off-axis near detector ND280 (right).

of iron/scintillator detectors, arranged to form a "cross" configuration, and two additional separate modules located at off-axis positions outside the main cross. An extra module, called the Proton Module, which consists of only scintillator bars, is located at the beam center between the horizontal and vertical standard modules to separate the neutrino interaction channels by detecting the protons and pions together with the muons from neutrino interactions. The center of the cross corresponds to the neutrino beam center, defined as $0^{\circ}$ with respect to the direction of the primary proton beamline. INGRID provides high-statistics monitoring of the neutrino beam intensity, direction, profile, and stability.

ND280 consists of a number of sub-detectors installed inside the refurbished UA1/NOMAD magnet that provides a magnetic field of 0.2 T. The Side Muon Range Detectors (SMRD) are installed in the magnet yoke, to track high angle muons, charged particles coming from neutrino interactions in the surrounding material upstream the detector and cosmic rays. Inside the magnet there is a $\pi^{0}$ detector ( $\mathrm{P} \emptyset \mathrm{D})$ to measure neutrino interactions with the production of $\pi^{0}$ in the final state on a water target and a tracker. The tracker consists of three Time Projection Chambers (TPC1, 2, 3) alternated with two one-tonne Fine-Grained Detectors (FGD1, 2). The TPCs are used to perform 3D tracking and to determine charge, momentum and energy loss by each charged particle traversing the detectors, while FGDs provide a neutrino mass target but also they have some tracking capabilities for charged particles coming from the interaction vertex. The $\mathrm{P} \emptyset \mathrm{D}$ and the tracker are then surrounded by a set of Electromagnetic Calorimeters (ECal). The primary goal of ND280 is to measure the flavor composition of the neutrino flux where is expected not to be affected by oscillation, to provide strong constraint on the predicted neutrino interaction rate in the far detector Super-Kamiokande where the neutrino oscillation is expected.

The understanding of neutrino interactions is essential to reduce the related uncertainties in the os- 
cillation analyses. In these proceedings, we report on new measurements of charged current (CC) inclusive and exclusive $v_{\mu}$ interactions in both INGRID and ND280 near detectors of T2K.

\section{2. $v_{\mu} \mathrm{CCO} \pi$ cross section measurements on carbon with ND280}

The $v_{\mu}$ CC Quasi-Elastic (CCQE) interaction $\left(v_{\mu}+n \rightarrow \mu^{-}+p\right)$ is categorized by the presence of a muon and a proton in the final state. However the protons are often very low momentum and cannot be seen in ND280 (if they even escape the nucleus). Therefore we define the CCQE-like topology as $\mathrm{CC} 0 \pi$, i.e. actively rejecting events with pions in the final state.

$v_{\mu} \mathrm{CC} 0 \pi$ interactions are measured using two different approaches. The first approach is based on the selection of $v_{\mu}$ CC events with one track required to be forward going, negatively charged and with a energy loss in the middle TPC (TPC2) compatible with that on a muon. The vertex of such a track should be inside upstream FGD (FGD1) and no reconstructed pions in the final state neither in FGD1 nor in TPC2. No explicit cuts are applied to include or exclude a proton track. The resulting selection has an efficiency of $50 \%$ with a purity of $70 \%$. The integrated flux-averaged cross section (Fig. 2) is obtained by using the Bayesian unfolding method. It is: $\left\langle\sigma_{v_{\mu} C C 0 \pi}\right\rangle_{\phi}=(4.06 \pm 0.757$ (stat. + syst. $\left.)\right) \times 10^{-39} \mathrm{~cm}^{2}$ nucleon.

The second approach uses a different set of selection criteria and a different method to extract

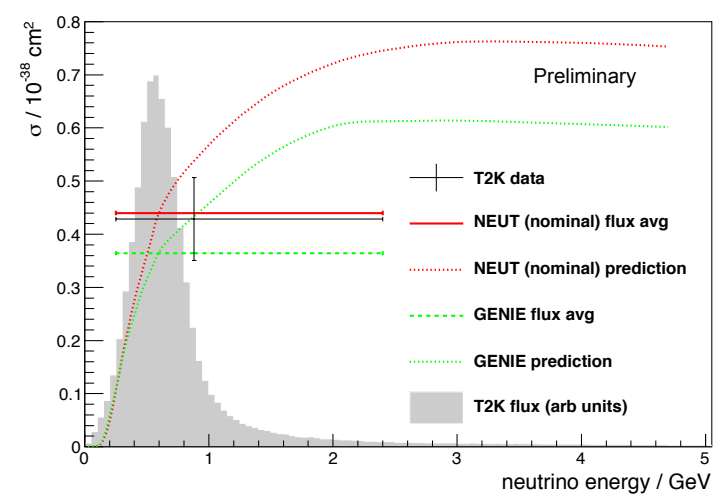

Figure 2: Total $v_{\mu} \mathrm{CC} 0 \pi$ cross section compared to the predictions of both NEUT [2] and GENIE [3] generators.

the cross section measurement. $v_{\mu} \mathrm{CC} 0 \pi$ events are selected asking for a track compatible with a muon, and possibly a track compatible with a proton, in the FGD1 or in the TPC2, with no other tracks in the final state. In this case, the selection covers a larger phase space with respect to the previous analysis, including also high angle muon candidates. This set of selection criteria leads to an efficiency of $40 \%$ with a purity of $90 \%$. The integrated flux-averaged cross section is obtained by using the maximum likelihood fit method. The likelihood fit is performed simultaneously on the selected sample and two control samples to constrain the backgrounds due to resonant pion production and Deep Inelastic Scattering. The resulting integrated flux-averaged cross section is: $\left\langle\sigma_{v_{\mu} C C 0 \pi}\right\rangle_{\phi}=(4.17 \pm 0.05$ (stat.) \pm 0.47 (syst.) $) \times 10^{-39} \mathrm{~cm}^{2}$ per nucleon. Both analyses give a reasonable agreement with the predictions of both NEUT and GENIE generators. Furthermore the agreement between these two different techniques is a strong evidence that the results are 
model independent. In Fig. 3 are shown the results obtained in the region $0.7<\cos (\theta)<0.8$ for different models. As can be seen from the left plot, data seems to prefer Random Phase Approximation (RPA) combined with Meson Exchange Current (MEC) models over RPA model alone. This analysis will be extended in the near future by using the muon and proton kinematics together,
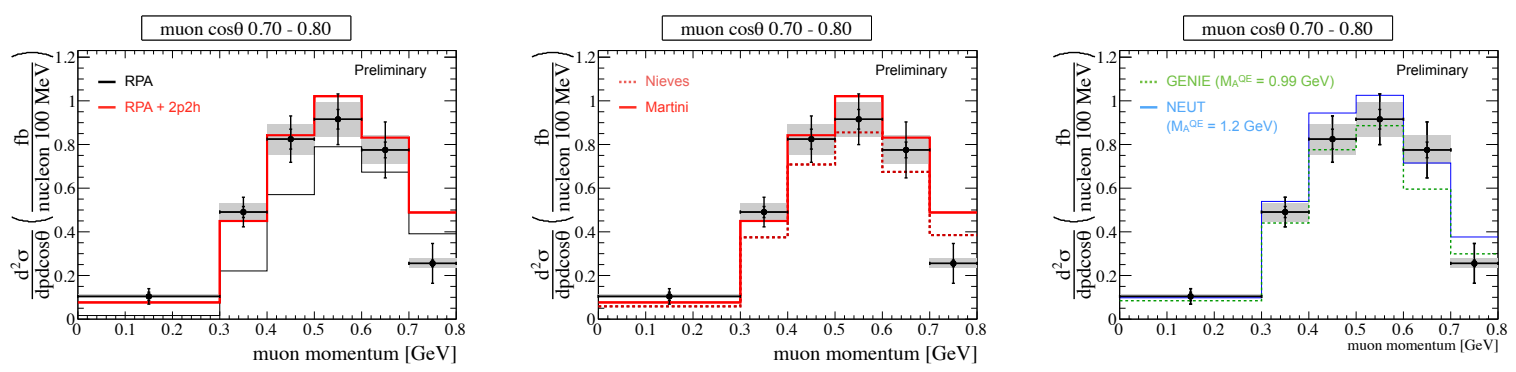

Figure 3: Measured cross-section as a function of muon momentum for the $0.7<\cos (\theta)<0.8$ bin compared to Monte Carlo models. Black dots are results from data with shape uncertainties: systematics and statistical uncertainties are outer bars while the only systematic uncertainty is shown in the inner bars. The gray band is the normalization uncertainty due to flux. In the left plot, red solid line is the prediction from Martini model [5], while black solid line is the prediction from Martini model without $2 \mathrm{p} 2 \mathrm{~h}$ contribution. In the central plot, red solid line is the prediction from Martini model, while red dotted line is the prediction from Nieves model [6]. In the right plot, blue solid line is the NEUT prediction $\left(M_{A}^{Q E}=1.21 \mathrm{GeV}\right)$, while green dotted line is the GENIE prediction $\left(M_{A}^{Q E}=0.99 \mathrm{GeV}\right)$.

combining the results on carbon and water to study the Final State Interaction (FSI) effects and combining neutrino and anti-neutrino data to unequivocally identify MEC effects.

\section{3. $v_{\mu}$ CCQE cross section measurement on Carbon with INGRID}

$v_{\mu} \mathrm{CCQE}$ interactions with carbon are identified by selecting events with either one (if only the $\mu^{-}$candidate is reconstructed) or two reconstructed tracks (the $\mu^{-}$and the proton candidates) coming from the same vertex in the Proton Module. The CCQE cross-section is calculated from the number of selected CCQE candidate events by subtracting background and correcting for selection efficiency at $1.94 \mathrm{GeV}$ and $0.93 \mathrm{GeV}$ mean energies. They are:

$\left\langle\sigma_{v_{\mu} C C Q E}\right\rangle_{1.94 \mathrm{GeV}}=\left(11.95 \pm 0.19\right.$ (stat.) ${ }_{-1.49}^{+1.80}$ (syst. $\left.)\right) \times 10^{-39} \mathrm{~cm}^{2}$ per nucleon,

$\left\langle\sigma_{v_{\mu} C C Q E}\right\rangle_{0.93 \mathrm{GeV}}=\left(10.64 \pm 0.37\right.$ (stat.) ${ }_{-1.65}^{+2.03}$ (syst. $\left.)\right) \times 10^{-39} \mathrm{~cm}^{2}$ per nucleon.

This cross section analysis was performed using three different neutrino interaction models: relativistic Fermi gas models with and without multinucleon interactions, and a spectral function model without multi-nucleon interactions [10]. Although these results are compatible with the model predictions, we found that the CCQE cross section results are dependent on the nuclear model and the existence of multi-nucleon interactions at the $10 \%$ level.

\section{4. $v_{\mu} \mathrm{CC} 1 \pi^{+}$cross sections measurement on water with ND280}

The $\mathrm{CC} 1 \pi^{+}$category includes all the $v_{\mu} \mathrm{CC}$ reactions producing a $\pi^{+}$in the final state. The measurement of the $v_{\mu} \mathrm{CC} 1 \pi^{+}$cross section on water, is performed using the downstream FGD 
(FGD2), which is composed of a sandwich of scintillators and water. $v_{\mu} \mathrm{CC}$ interactions are selected with a vertex inside the first layer downstream of the water modules of FGD2 to select a water-enhanced sample. Furthermore only events with only one positive pion and any number of protons are taken into account. The selection efficiency is $13.1 \%$ with a purity of $50.6 \%$. The integrated flux-averaged $\mathrm{CC} 1 \pi^{+}$cross section is obtained by using the Bayesian unfolding method. The result is: $\left\langle\sigma_{v_{\mu} C C 1 \pi^{+}}\right\rangle_{\phi}=(7.778 \pm 1.115$ (stat.) \pm 2.920 (syst.) $) \times 10^{-40} \mathrm{~cm}^{2}$ per nucleon. As can be seen from Fig. 4 , this result shows a $1 \sigma$ suppression in the $\mathrm{CC} 1 \pi^{+}$cross section if compared to the NEUT prediction and it is worse if compared with the GENIE prediction. An improved analysis with more data is foreseen in the future in order to reduce the current large uncertainties on this measurement.

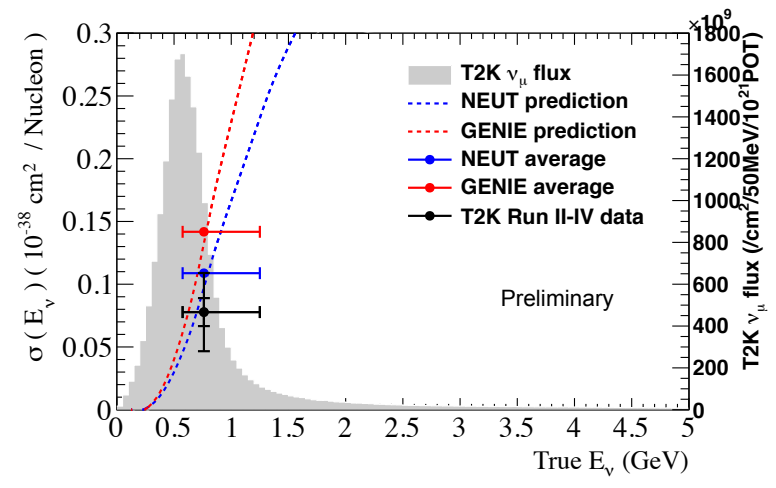

Figure 4: Total $v_{\mu} \mathrm{CC} 1 \pi^{+}$cross section on water. The T2K data point is placed at the $v_{\mu}$ flux mean energy. The vertical error represents the total uncertainty, and the horizontal bar represents $68 \%$ of the flux each side of the mean. The T2K flux distribution is shown in gray. The NEUT and GENIE predictions are the total $v_{\mu}$ $\mathrm{CC} 1 \pi^{+}$predictions as a function of neutrino energy. The NEUT and GENIE averages are the flux-averaged predictions.

\section{5. $v_{\mu}$ CC Coherent cross sections measurement on carbon with ND280}

The $v_{\mu}$ induced CC coherent pion production on Carbon $\left(v_{\mu}+{ }^{12} C \rightarrow \mu^{-}+\pi^{+}+{ }^{12} C\right)$ was studied at a mean neutrino energy of $0.86 \mathrm{GeV}$. These kind of interactions are selected with a cut-based analysis which identifies events with only a reconstructed $\mu^{-}$and a reconstructed $\pi^{+}$coming from the same vertex inside the FGD1 and with a low amount of energy deposited close to the vertex. The CC Coherent process is limited to low $|t|$, where $t$ is defined to be the squared four-momentum transfer to the nucleus $\left(|t|=\left(q-p_{\pi^{+}}\right)^{2}=\left|\left(\left(p_{v}-p_{\mu^{-}}\right)-p_{\pi^{+}}\right)\right|\right)$. A $2.7 \sigma$ excess of events was found above the background prediction at low $|t|$. Two models are used to determine the selection efficiency: the Rein-Sehgal model [7] in GENIE, and the microscopic Alvarez-Ruso model [8]. The resulting flux-averaged cross section are respectively $\left\langle\sigma_{v_{\mu} C C C o h}\right\rangle_{R S}=\left(4.2 \pm 1.0\right.$ (stat.) ${ }_{-1.4}^{+1.5}$ (syst. $\left.)\right) \times 10^{-40} \mathrm{~cm}^{2}$ per nucleon, and $\left\langle\sigma_{v_{\mu} C C C o h}\right\rangle_{A R}=$ $\left(3.6 \pm 0.8\right.$ (stat.) ${ }_{-1.3}^{+1.4}$ (syst.) $) \times 10^{-40} \mathrm{~cm}^{2}$ per nucleon. As can be seen from Fig. 6 , the combined effect of the statistical uncertainty in the data with the main systematic uncertainty related to background simulation, prevent any statement on which model is preferred by the data. 


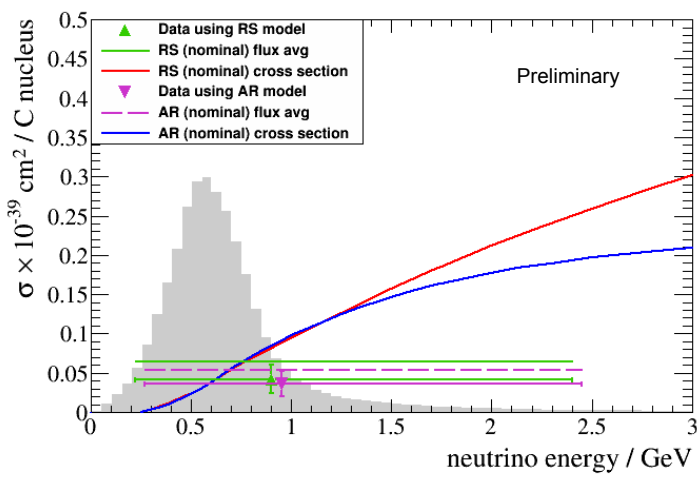

Figure 5: Comparison of the results from each coherent model. The central value in energy for the AlvarezRuso model has been shifted to the right by $0.05 \mathrm{GeV}$ to enhance clarity. The solid and dashed horizontal lines encompass the central $90 \%$ of the neutrino flux observed by ND280.

\section{6. $v_{\mu} \mathrm{CC}$ inclusive cross section measurement on iron and hydrocarbon with INGRID}

The $v_{\mu}$ CC interactions with hydrocarbon are selected by identifying the $\mu^{-}$candidate with the vertex in the Proton Module, while the $v_{\mu}$ CC interactions with iron, are selected by identifying the $\mu^{-}$candidate with the vertex in the horizontal central standard module. The flux-averaged $v_{\mu}$ $\mathrm{CC}$ inclusive cross section on hydrocarbon and iron are calculated from the number of selected events using the background subtraction and efficiency correction at a mean neutrino energy of $1.51 \mathrm{GeV}$. They are: $\sigma_{C C}^{C H}=\left(1.379 \pm 0.009\right.$ (stat.) ${ }_{0.147}^{0.178}$ (syst.) $) \times 10^{-38} \mathrm{~cm}^{2}$ per nucleon, and $\sigma_{C C}^{F e}=$ $\left(1.444 \pm 0.002\right.$ (stat.) ${ }_{0.157}^{0.189}$ (syst.) $) \times 10^{-38} \mathrm{~cm}^{2}$ per nucleon. As can be seen from Fig. 6, the results show a good agreement with the predictions of both NEUT and GENIE generators. Moreover in [9] also the cross section ratio $\sigma_{C C}^{F e} / \sigma_{C C}^{C H}$ is reported, showing also in this case a good agreement with the predictions.
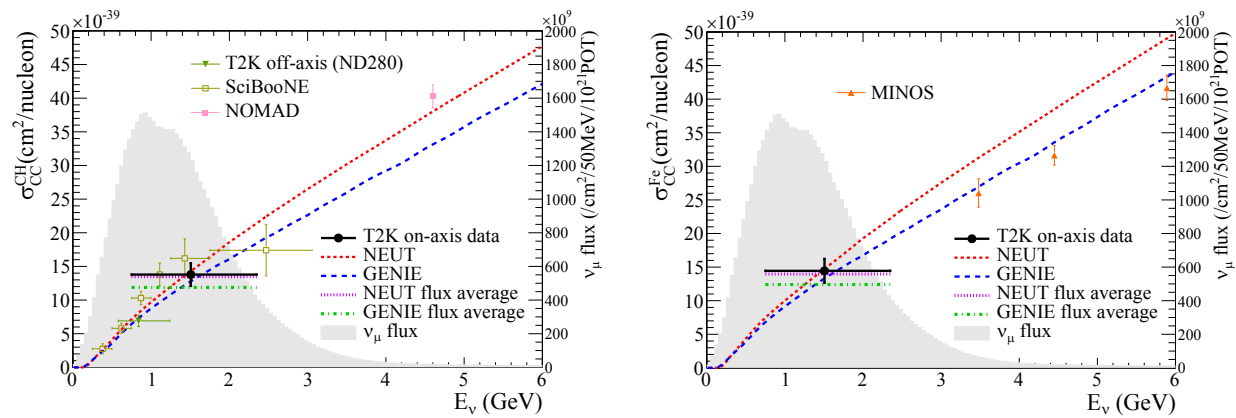

Figure 6: The inclusive $v_{\mu} \mathrm{CC}$ cross section on $\mathrm{CH}$ (left) and that on Fe (right) with predictions by NEUT and GENIE. The isoscalar corrections are not applied to our data or predictions. Our data point is placed at the flux mean energy. The vertical error bar represents the total (statistical and systematic) uncertainty, and the horizontal bar represents $68 \%$ of the flux at each side of the mean energy. 


\section{Conclusions}

The T2K experiment has performed many neutrino cross section measurements at the near detector complex. All the new cross section results are in agreement with the predictions of the neutrino models except the $v_{\mu} \mathrm{CC} 1 \pi^{+}$cross sections measurement on water, that shows a $1 \sigma$ suppression if compared to the NEUT prediction. However, an improved analysis of the $\mathrm{CC} 1 \pi^{+}$cross section on water is foreseen in the future in order to reduce the large uncertainties. Furthermore we expect to release soon also anti-neutrino cross section measurements that will help our understanding of neutrino interactions.

\section{References}

[1] K. Abe et al. (T2K Collaboration), Nucl. Instrum. Methods A659, 106 (2011).

[2] Y. Hayato, ActaPhys. Pol. B40, 2477 (2009).

[3] C. Andreopoulos et al., Nucl. Instrum. Methods Phys. Res., Sec. A 614, 87 (2010).

[4] K.Abe et al. (T2K Collaboration), Phys. Rev. Lett. 113, 241803 (2014).

[5] M. Martini, M. Ericson, G. Chanfray, J. Marteau, Physical Review C80, 065501 (2009).

[6] Nieves, I. Ruiz Simo, and M.J. Vicente Vacas, Phys. Lett. B707, $72-75$ (2012).

[7] D. Rein and L.M. Sehgal. Nuclear Physics B, Volume 223, p29-44, 1983.

[8] L. Alvarez-Ruso et al. Physical Review C75, p055501 (2007).

[9] K. Abe et al. (T2K Collaboration), Phys. Rev. D90, 052010 (2014).

[10] K. Abe et al. (T2K Collaboration), Phys. Rev. D91, 112002 (2015). 